\title{
Trabeculectomy is not the best surgical option for glaucoma
}

PK Wishart

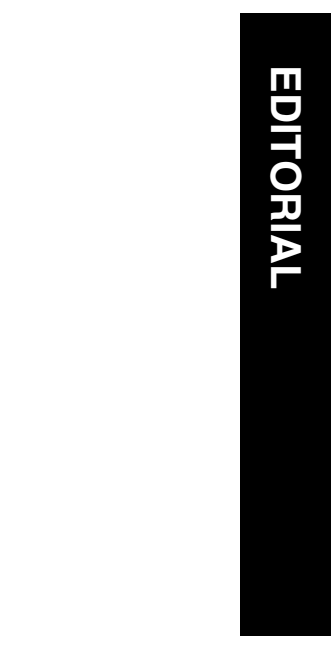

Eye (2008) 22, 603-606; doi:10.1038/eye.2008.73; published online 14 March 2008

The goal of glaucoma surgery should be the preservation of visual function, the avoidance of sight-threatening complications, and the preservation of the quality of life of the patient. Curiously, the majority of glaucoma surgeons still consider trabeculectomy the best surgical option for their patients, despite evidence that trabeculectomy cannot meet these goals.

The trabeculectomy operation has already been abandoned in favour of the augmented trabeculectomy with the antimetabolite mitomycin-C (MMC) ${ }^{1}$ by the majority of UK and US glaucoma specialists. ${ }^{2} \mathrm{MMC}$ trabeculectomy, however, is not safe. The risk of bleb-related endophthalmitis (BRE) ranges from $1.5 \%$ per patient year ${ }^{3}$ to $10 \%$ in one year, ${ }^{4}$ with 11 recent studies giving an average incidence of $2.8 \%$ per year. ${ }^{5-13}$ As the mean follow-up of these 11 studies was 19 months, the true incidence may be higher, as institutional reviews of BRE show that the mean time of presentation of BRE is 32 months postoperatively. ${ }^{14-18}$

Of equal importance as a cause of central visual loss after MMC trabeculectomy is hypotony maculopathy, with a mean incidence of $3.2 \%$ per annum reported in 7 recent studies (mean follow-up of 28 months). ${ }^{4,9,19-23}$ The Collaborative Initial Glaucoma Treatment Study (CIGTS) showed that trabeculectomy increases the risk of cataract formation eightfold in the first postoperative year and by fourfold over 5 years. ${ }^{24}$ Cataracts can be removed, but bleb failure occurs in $22 \%$ of eyes as a consequence of cataract surgery. ${ }^{25}$ Numerous papers are published advising techniques for increasing the safety of MMC trabeculectomy, with posterior application of MMC and strategies to avoid early postoperative hypotony using tight sutures that can later be released, lasered, or adjusted. ${ }^{26}$ However, these postoperative manipulations can cause serious complications, such as endophthalmitis, bleb leaks, suprachoroidal haemorrhage, flat anterior chambers, ${ }^{15,27,28}$ and may reduce the success of the operation. ${ }^{29}$

A 2006 study described a 'Safe trabeculectomy technique' that had a $1.5 \%$ rate of BRE in the first year. ${ }^{13}$ A recent editorial ${ }^{30}$ on the Tube $v s$ Trabeculectomy (TVT) study ${ }^{31}$ described as 'excellent' the first year's results of MMC trabeculectomy despite persistent hypotony causing $3 \%$ of failures, $1 \%$ of eyes suffering BRE, and an additional $2 \%$ of cases suffering blebitis, ${ }^{32}$ a precursor of endophthalmitis. ${ }^{14}$ Surely such a high incidence of serious bleb-related complications is at odds with the description of an 'excellent record'?

As to efficacy, MMC trabeculectomy in the TVT study had a $13.5 \%$ failure rate and a $57 \%$ incidence of postoperative complications, including a $10 \%$ incidence of shallow or flat anterior chamber, $3 \%$ hypotony maculopathy, $3 \%$ suprachoroidal haemorrhage, and 19\% choroidal effusions. ${ }^{31,32}$ There were 85 surgically invasive postoperative interventions including $49 \%$ laser suture lysis and $22 \%$ needling. ${ }^{31}$ Two recent studies of MMC trabeculectomy from Europe showed similar rates of postoperative interventions and complications. ${ }^{12,13}$ Can an operation that requires such a large number of secondary invasive procedures be considered effective? Are our glaucoma patients not entitled to expect to go through the trauma and anxiety of surgery only once? Is such an approach an appropriate use of the talent of glaucoma specialists and the limited economic resources available? The high incidence of bleb dysaesthesia following MMC trabeculectomy present in up to $64 \%$ of eyes ${ }^{33}$ is surely unacceptable, especially as pain and watering are also the symptoms of blebitis, which the patient has been instructed to report immediately ${ }^{34}$ lest blebitis progresses to endophthalmitis.

In glaucoma, the EMGT ${ }^{35}$ has shown that for each 1-mm Hg lowering of the intraocular
St Paul's Eye Unit, Royal Liverpool University Hospital, Liverpool, UK

Correspondence: PK Wishart, St Paul's Eye Unit, Royal Liverpool University Hospital,

Prescot Street, Liverpool Merseyside L7 8XP, UK Tel: + 0044 (0)151706 3968;

Fax: + 0044 (0) 151706 5861.

E-mail: peter.wishart@ rlbuht.nhs.uk

Presented in 'The Great Debate' at the Royal College of Ophthalmologists Annual Conference, May 2007, Birmingham, UK 
pressure (IOP), the risk of progression of visual field loss decreases by $10 \%$. Many surgeons extrapolate this and the findings of the Advanced Glaucoma Intervention (AGIS) ${ }^{36}$ study to justify their belief that glaucoma patients require a low IOP to preserve vision, forgetting that for every 3-mm Hg reduction in IOP posttrabeculectomy, the risk of endophthalmitis rises 50-fold. ${ }^{37}$ In fact, the EMGT showed that despite IOP reduction, $45 \%$ of treated eyes still showed progression ${ }^{35}$ and the AGIS investigators point out that their data shows '... it is clear that maintaining IOP $<18 \mathrm{~mm} \mathrm{Hg}$ does not ensure the preservation of the visual field' as $15 \%$ of eyes in the group with IOP $<18 \mathrm{~mm} \mathrm{Hg}$ at all visits showed progression. ${ }^{36}$ Furthermore, reanalysis of the AGIS data by pointwise linear regression analysis showed no relation between mean IOP and visual field loss progression. ${ }^{38}$ Parc et al reported that $46 \%$ of posttrabeculectomy eyes that went blind had an IOP of $14 \mathrm{~mm} \mathrm{Hg}$ compared to a mean of $15.4 \mathrm{~mm} \mathrm{Hg}$ in those eyes not going blind. ${ }^{39}$ CIGTS showed that despite a $48 \%$ reduction in IOP in the trabeculectomy eyes, there was more visual field loss progression in this group than that occurred in the medically treated eyes, which only had a 35\% reduction in IOP. ${ }^{40}$ Even in the Moorfields' Primary Treatment Trial, when fields were tested with automated perimetry, there was no difference in progression of field loss between the medically treated eyes (mean IOP of $19 \mathrm{~mm} \mathrm{Hg}$ ) and the trabeculectomy eyes (mean IOP of $14 \mathrm{~mm} \mathrm{Hg}$ ). ${ }^{41}$ As yet there are no studies showing that low IOP achieved by MMC trabeculectomy confers preservation of visual field. Indeed, one study ${ }^{9}$ showed that the worst field loss progression occurred in eyes which underwent MMC trabeculectomy, despite having the lowest IOP. Primary open angle glaucoma only has a $3 \%$ per annum risk of a measurable decline in visual field threshold ${ }^{42}$ yet the risk of blindness from MMC trabeculectomy is 6\% per annum from a combination of BRE $(2.8 \%)$ and hypotony maculopathy (3.2\%).

The alternative to trabeculectomy is the operation of viscocanalostomy (VC), which provides bleb-free drainage surgery. Every VC involves performing a deep sclerectomy (DS) to identify and then de-roof the canal of Schlemm- creating an '-ostomy' in the canal. Those who call the operation a DS tend to use an implant to encourage formation of an intrascleral lake, ${ }^{43}$ whereas those calling the procedure a VC use viscoelastic and tight closure of the superficial scleral flap, to encourage aqueous to drain into the cut ends of Schlemm's canal. ${ }^{44}$ Published randomized controlled trials show no statistically significant difference in IOP lowering with trabeculectomy compared to VC. ${ }^{45-48}$ These studies have all shown that trabeculectomy has a significantly higher complication rate of shallow ACs and cataract formation. ${ }^{45-48}$ There are now seven long-term studies (mean 4 years follow-up) involving more than 1000 eyes that have undergone VC/DS without a single case of endophthalmitis or hypotony being reported. ${ }^{43,44,49-53}$ Few long-term studies exist for MMC trabeculectomy, but with a 23\% 5-year incidence of sight-threatening bleb-related complications ${ }^{3}$ and $3 \%$ per annum hypotony maculopathy, some 300 eyes might be expected to have suffered from these complications had MMC

trabeculectomy been performed on these eyes rather than VC.

For the patient, freedom from the discomfort of a filtering bleb and the worry of developing BRE represent major quality of life improvements over trabeculectomy.

When combined with phacocataract extraction, VC is highly effective. The complete success rate (CSR) (IOP $\leqslant 18 \mathrm{~mm} \mathrm{Hg}$ without medication) following phacoVC in one study of 165 eyes was $50 \%$ at 3 years postoperatively, ${ }^{53}$ whereas the CSR reported in a recent study of 173 eyes undergoing phacotrabeculectomy (102 with MMC) was $18.5 \%$ at 3 years postoperatively. ${ }^{29} \mathrm{VC}$ has also proved effective in cases of glaucoma secondary to uveitis or previous failed trabeculectomy. ${ }^{54}$

In an eye with an obliterated angle through a disease such as neovascular glaucoma, VC would not be possible and a glaucoma drainage device would be the operation of choice rather than a trabeculectomy. The TVT study showed that tubes have equal success but cause significantly less complications than MMC trabeculectomy. ${ }^{31,32}$ The evidence shows that MMC trabeculectomy is neither safe nor effective. On the basis of the evidence, surely glaucoma surgeons should adopt $\mathrm{VC} / \mathrm{DS}$ and abandon trabeculectomy?

\section{References}

1 Franks W. Trypan blue staining of antiproliferative agents for trabeculectomy surgery and bleb needling. $\mathrm{Br} \mathrm{J}$ Ophthalmol 2005; 89: 1394-1395.

2 Liebmann JM, Ritch R. Bleb related ocular infection: a feature of the HELP syndrome. Hypotony, endophthalmitis, leak, pain. Br J Ophthalmol 2000; 84: 1338-1339.

3 DeBry PW, Perkins TW, Heatley G, Kaufman P, Brumback LC. Incidence of late-onset bleb-related complications following trabeculectomy with mitomycin. Arch Ophthalmol 2002; 120: 297-300.

4 Mietz H, Krieglstein GK. Suramin to enhance glaucoma filtering procedures: a clinical comparison with mitomycin. Ophthalmic Surg Lasers 2001; 32: 358-369.

5 Megevand GS, Salmon JF, Scholtz RP, Murray AD. The effect of reducing the exposure time of mitomycin $\mathrm{C}$ in glaucoma filtering surgery. Ophthalmology 1995; 102: 84-90.

6 Greenfield DS, Suner IJ, Miller MP, Kangas TA, Palmberg PF, Flynn Jr HW. Endophthalmitis after filtering surgery with mitomycin. Arch Ophthalmol 1996; 114: 943-949. 
7 Akarsu C, Onol M, Hasanreisoglu B. Effects of thick Tenon's capsule on primary trabeculectomy with mitomycin-C. Acta Ophthalmol Scand 2003; 81: 237-241.

8 Beck AD, Wilson WR, Lynch MG, Lynn MJ, Noe R. Trabeculectomy with adjunctive mitomycin $\mathrm{C}$ in pediatric glaucoma. Am J Ophthalmol 1998; 126: 648-657.

9 Membrey WL, Bunce C, Poinoosawmy DP, Fitzke FW, Hitchings RA. Glaucoma surgery with or without adjunctive antiproliferatives in normal tension glaucoma: 2 visual field progression. $\mathrm{Br} \mathrm{J}$ Ophthalmol 2001; 85: 696-701.

10 Wells AP, Cordeiro MF, Bunce C, Khaw PT. Cystic bleb formation and related complications in limbus- vs fornixbased conjunctival flaps in pediatric and young adult trabeculectomy with mitomycin C. Ophthalmology 2003; 110 2192-2197.

11 Belyea DA, Dan JA, Lieberman MF, Stamper RL. Midterm follow-up results of combined phacoemulsification, lens implantation, and mitomycin-C trabeculectomy procedure. J Glaucoma 1997; 6: 90-98.

12 Alwitry A, Patel V, King AW. Fornix vs limbal-based trabeculectomy with mitomycin C. Eye 2005; 19: 631-636.

13 Stalmans I, Gillis A, Lafaut AS, Zeyen T. Safe trabeculectomy technique: long term outcome. Br J Ophthalmol 2006; 90: 44-47.

14 Lehmann OJ, Bunce C, Matheson MM, Maurino V, Khaw PT, Wormald R et al. Risk factors for development of post-trabeculectomy endophthalmitis. Br J Ophthalmol 2000; 84: 1349-1353.

15 Song A, Scott IU, Flynn Jr HW, Budenz DL. Delayed-onset bleb-associated endophthalmitis: clinical features and visual acuity outcomes. Ophthalmology 2002; 109: 985-991.

16 Waheed S, Ritterband DC, Greenfield DS, Liebmann JM, Seedor JA, Ritch R. New patterns of infecting organisms in late bleb-related endophthalmitis: a ten year review. Eye 1998; 12(Part 6): 910-915.

17 Busbee BG, Recchia FM, Kaiser R, Nagra P, Rosenblatt B, Pearlman RB. Bleb-associated endophthalmitis: clinical characteristics and visual outcomes. Ophthalmology 2004; 111: 1495-1503.

18 Ciulla TA, Beck AD, Topping TM, Baker AS. Blebitis, early endophthalmitis, and late endophthalmitis after glaucomafiltering surgery. Ophthalmology 1997; 104: 986-995.

19 Manners T, Salmon JF, Barron A, Willies C, Murray AD. Trabeculectomy with mitomycin $\mathrm{C}$ in the treatment of posttraumatic angle recession glaucoma. Br J Ophthalmol 2001; 85: $159-163$

20 Scott IU, Greenfield DS, Schiffman J, Nicolela MT, Rueda JC, Tsai JC et al. Outcomes of primary trabeculectomy with the use of adjunctive mitomycin. Arch Ophthalmol 1998; 116: 286-291.

21 Lopes JF, Moster MR, Wilson RP, Altangerel U, Alvim HS, Tong MG et al. Subconjunctival sodium hyaluronate $2.3 \%$ in trabeculectomy: a prospective randomized clinical trial. Ophthalmology 2006; 113: 756-760.

22 Bindlish R, Condon GP, Schlosser JD, D'Antonio J, Lauer KB, Lehrer R. Efficacy and safety of mitomycin-C in primary trabeculectomy: five-year follow-up. Ophthalmology 2002; 109: 1336-1341.

23 Cashwell LF, Martin CA. Axial length decrease accompanying successful glaucoma filtration surgery. Ophthalmology 1999; 106: 2307-2311.

24 Musch DC, Gillespie BW, Niziol LM, Janz NK, Wren PA, Rockwood EJ et al. Cataract extraction in the collaborative initial glaucoma treatment study: incidence, risk factors, and the effect of cataract progression and extraction on clinical and quality-of-life outcomes. Arch Ophthalmol 2006; 124: 1694-1700.

25 Chen PP, Weaver YK, Budenz DL, Feuer WJ, Parrish RK. Trabeculectomy function after cataract extraction. Ophthalmology 1998; 105: 1928-1935.

26 Wells AP, Bunce C, Khaw PT. Flap and suture manipulation after trabeculectomy with adjustable sutures: titration of flow and intraocular pressure in guarded filtration surgery. J Glaucoma 2004; 13: 400-406.

27 Burchfield JC, Kolker AE, Cook SG. Endophthalmitis following trabeculectomy with releasable sutures. Arch Ophthalmol 1996; 114: 766.

28 Macken P, Buys Y, Trope GE. Glaucoma laser suture lysis Br J Ophthalmol 1996; 80: 398-401.

29 Banitt M, Juzych MS, Chopra V, Hughes BA, Kim C. The effect of the timing of scleral flap suture release on the safety and long-term success of phacotrabeculectomy. Am I Ophthalmol 2006; 141: 742-744.

30 Jamil AL, Mills RP. Glaucoma tube or trabeculectomy? that is the question. Am J Ophthalmol 2007; 143: 141-142.

31 Gedde SJ, Schiffman JC, Feuer WJ, Herndon LW, Brandt JD, Budenz DL. Treatment outcomes in the tube vs trabeculectomy study after one year of follow-up. Am J Ophthalmol 2007; 143: 9-22.

32 Gedde SJ, Herndon LW, Brandt JD, Budenz DL, Feuer WJ, Schiffman JC. Surgical complications in the Tube vs Trabeculectomy Study during the first year of follow-up. Am J Ophthalmol 2007; 143: 23-31.

33 Budenz DL, Hoffman K, Zacchei A. Glaucoma filtering bleb dysesthesia. Am J Ophthalmol 2001; 131: 626-630.

34 Mac I, Soltau JB. Glaucoma-filtering bleb infections. Curr Opin Ophthalmol 2003; 14: 91-94.

35 Heijl A, Leske MC, Bengtsson B, Hyman L, Bengtsson B, Hussein M. Reduction of intraocular pressure and glaucoma progression: results from the Early Manifest Glaucoma Trial. Arch Ophthalmol 2002; 120: 1268-1279.

36 The AGIS Investigators. The Advanced Glaucoma Intervention Study (AGIS): 7. The relationship between control of intraocular pressure and visual field deterioration. Am J Ophthalmol 2000; 130: 429-440.

37 Jampel HD, Quigley HA, Kerrigan-Baumrind LA, Melia BM, Friedman D, Barron Y. Risk factors for late-onset infection following glaucoma filtration surgery. Arch Ophthalmol 2001; 119: 1001-1008.

38 Nouri-Mahdavi K, Hoffman D, Coleman AL, Liu G, Li G, Gaasterland D et al. Predictive factors for glaucomatous visual field progression in the Advanced Glaucoma Intervention Study. Ophthalmology 2004; 111: 1627-1635.

39 Parc CE, Johnson DH, Oliver JE, Hattenhauer MG, Hodge DO. The long-term outcome of glaucoma filtration surgery. Am J Ophthalmol 2001; 132: 27-35.

40 Lichter PR, Musch DC, Gillespie BW, Guire KE, Janz NK, Wren PA et al. Interim clinical outcomes in the Collaborative Initial Glaucoma Treatment Study comparing initial treatment randomized to medications or surgery. Ophthalmology 2001; 108: 1943-1953.

41 Hitchings RA, Migdal CS, Wormald R, Poinooswamy D, Fitzke F. The primary treatment trial: changes in the visual field analysis by computer-assisted perimetry. Eye 1994; 8(Part 1): 117-120. 
42 Smith SD, Katz J, Quigley HA. Analysis of progressive change in automated visual fields in glaucoma. Invest Ophthalmol Vis Sci 1996; 37: 1419-1428.

43 Shaarawy T, Nguyen C, Schnyder C, Mermoud A. Comparative study between deep sclerectomy with and without collagen implant: long term follow up. $\mathrm{Br}$ J Ophthalmol 2004; 88: 95-98.

44 Stegmann R, Pienaar A, Miller D. Viscocanalostomy for open-angle glaucoma in black African patients. J Cataract Refract Surg 1999; 25: 316-322.

45 Chiselita D. Non-penetrating deep sclerectomy vs trabeculectomy in primary open-angle glaucoma surgery. Eye 2001; 15: 197-201.

46 El Sayyad F, Helal M, El-Kholify H, Khalil M, El-Maghraby A Nonpenetrating deep sclerectomy vs trabeculectomy in bilateral primary open-angle glaucoma. Ophthalmology 2000; 107: 1671-1674.

47 O'Brart DP, Shiew M, Edmunds B. A randomised, prospective study comparing trabeculectomy with viscocanalostomy with adjunctive antimetabolite usage for the management of open angle glaucoma uncontrolled by medical therapy. Br J Ophthalmol 2004; 88: 1012-1017.

48 Carassa RG, Bettin P, Fiori M, Brancato R. Viscocanalostomy vs trabeculectomy in white adults affected by open-angle glaucoma: a 2-year randomized, controlled trial Ophthalmology 2003; 110: 882-887.

49 Lachkar Y, Neverauskiene J, Jeanteur-Lunel MN, Gracies H, Berkani M, Ecoffet $\mathrm{M}$ et al. Nonpenetrating deep sclerectomy: a 6-year retrospective study. Eur J Ophthalmol 2004; 14: 26-36.

50 Shaarawy T, Nguyen C, Schnyder C, Mermoud A. Five year results of viscocanalostomy. Br J Ophthalmol 2003; 87: 441-445.

51 Sunaric-Megevand G, Leuenberger PM. Results of viscocanalostomy for primary open-angle glaucoma. Am J Ophthalmol 2001; 132: 221-228.

52 Wishart PK, Wishart MS, Porooshani H. Viscocanalostomy and deep sclerectomy for the surgical treatment of glaucoma: a longterm follow-up. Acta Ophthalmol Scand 2003; 81: 343-348.

53 Wishart MS, Dagres E. Seven-year follow-up of combined cataract extraction and viscocanalostomy. J Cataract Refract Surg 2006; 32: 2043-2049.

54 Choudhary A, Wishart PK. Non-penetrating glaucoma surgery augmented with mitomycin $\mathrm{C}$ or 5-fluorouracil in eyes at high risk of failure of filtration surgery: long-term results. Clin Experiment Ophthalmol 2007; 35: 340-347. 